\title{
EUFEMISMO IN ITALIANO: INTERDIZIONE VERBALE NEL LESSICO LEGATO ALLA SESSUALITÀ FEMMINILE
}

\begin{abstract}
Beszterda Ingeborga, Szpingier Beata, Eufemismo in italiano: interdizione verbale nel lessico legato alla sessualità femminile [Euphemism in Italian: linguistic interdiction in the vocabulary concerning the feminine sexuality]. Studia Romanica Posnaniensia, Adam Mickiewicz University Press, Poznań, vol. XXXIII: 2006, pp. 219-226. ISBN 83-232-1643-6, ISSN 0137-2475.
\end{abstract}

The objective of this analysis is a description of euphemism in the onomastics referring to a woman through the lexicology of erotica found in everyday circulation. The description is not merely confined to lexemes referring to a women but also contains words describing parts of her body, physiological functions and sexual activity. Sexuality belongs to those domain which constitute a basis for a linguistics ban, indubitably, repression too. Studies of language prove that linguistic denial is inseparably tied with and present in interhuman communication.

1. Si identifica il fenomeno di eufemismo come l'insieme di produzioni linguistiche che permettono di evitare l'uso di parole o espressioni troppo aspre o volgari sostituendole con quelle più discrete, sinonimiche o perifrastiche. Alla base di un giro di parole stanno ragioni psicologiche: si sceglie un termine attenuato per scrupoli religiosi, morali o infine per rispetto delle convenienze sociali. Tale movente mentale, pur essendo un fatto extralinguistico, viene considerato in sede linguistica, perché esercita una diversa azione sulla lingua: il divieto obbliga il parlante ad omettere i termini che indicano direttamente una cosa vietata. Le cause che impediscono di pronunciare una data parola fanno sì che si scelgano espressioni molto generiche ossia, a volte, non si nomina in modo esplicito un enunciato interdetto (Galli de Paratesi, 1964: 7).

Diversi tipi di interdizione presentano molteplici aspetti linguistici e danno luogo a vari moduli di sostituzione che variano a seconda della classe sociale, dell'ambiente, del sesso e anche del momento storico (sul piano diacronico il rinnovamento all'interno del lessico eufemistico è dovuto in maggior parte al ricambio del vocabolario; sul piano sincronico la ricerca di nuove coperture determina la nascita di termini sinonimici tra i quali molti sono vezzeggiativi, affettivi o fantasiosi). 
Il discorso di ogni giorno è cosparso di eufemismi. Il carattere affettivo della parola esige la scelta conveniente che si adatta alla data situazione, così chi parla cerca di esprimersi con le forme accettabili dalla norma sociale (Widlak, 1970:23). La scelta dei sostituti dipende dal livello culturale degli utenti: usando termini eufemistici si rende più alto il linguaggio banale degli strati inferiori nei quali si nega l'utilizzazione eufemistica dei termini dotti o scientifici. Nel linguaggio popolare sono numerosi i vocaboli sostituiti per ineffabilità, per es. cose che ̀̀ meglio non dire, oppure si usano termini generali per es. andare con qualcuno 'aver rapporti sessuali'. Nel linguaggio comune delle persone colte si ricorre a sostituzioni non solo di parole dotte, ma anche a quelle delle lingue straniere (Galli de Paratesi, 1964: 24), per es. madama dal francese madame. Succede anzi agli utenti di questa classe elevata di introdurre nelle conversazioni espressioni volgari o familiari: succede che i gerghi di certi gruppi sociali si propaghino talvolta nel parlato di un'intera comunità come nel caso di sfiga e sfigato 'sfortuna, sfortunato'.

2. Nell'ambito della presente analisi si intende descrivere l'eufemismo nella onomastica femminile attraverso il lessico erotico di un registro piuttosto popolare il quale non riguarderà solo le parole che caratterizzano la donna, ma anche quelle che la riguardano come alcune parti del corpo, funzioni fisiologiche, rapporti con l'uomo, ecc... Ovviamente la categoria della sessualità si colloca tra quelle che stanno alla base dei fenomeni riguardanti l'interdizione linguistica e provoca la repressione più netta.

Gli studi profondi della lingua hanno condotto alla costatazione che essa è inseparabile dalla società e resta nell'uso dell'ambito quotidiano; serve a indicare fenomeni della vita sociale sia biologica che sentimentale, si differenzia a seconda del luogo d'abitazione e dell'appartenenza ad uno strato sociale. L'evoluzione della lingua resta in rapporto reciproco con l'evoluzjone della società. Non si può analizzare costumi, gruppi e le relazioni tra loro senza una storia della lingua. Le deformazioni e variazioni della parola forniscono indicazioni per la storia dei fatti sociali e culturali (Beccaria, 1988: 8).

A questo punto si deve sottolineare che da qualche anno la comunicazione all'interno della società diventa in misura sempre crescente "informatizzata". I modi, e pure i mezzi, di comunicazione come per esempio il telefono, il computer e dunque internet, ma anche lo sviluppo della rete cellulare ed il grande successo degli SMS limitano i rapporti amichevoli e quelli amorosi ad un livello molto modesto, per non dire povero, rispetto alla corrispondenza scritta il cui ruolo era veramente "onnipotente", adesso diminuisce sempre di più. La lingua dei giornali, del cinema diventa anche lei, più semplice perché orientata verso un pubblico vasto ed in generale non tanto istruito (dipende infatti dai periodici e da alcuni film di un cinema cosiddetto ambizioso) viene da una parte semplificata dall'altra si arricchisce di espressioni ricercate non sempre di un livello alto.

E poi, la lingua vive nuovi ritmi delle nuove generazioni. I linguaggi della tecnica hanno attualmente maggior peso. Lasciati i vecchi mezzi di locomozione (il 
cavallo e le vie d'acqua), la nostra è diventata una società motorizzata, il che ha intensificato i traslati automobilistici nel linguaggio colloquiale e riguarda anche le designazioni femminili, ad esempio: carrozzeria per corpo di donna (Beccaria, 1988: 103-104).

3. Il vocabolario di cui ci si serve per descrivere la donna nei rapporti amichevoli o intimi, oppure i rapporti amichevoli stessi, i pensieri, i sentimenti, le dichiarazioni si differenziano secondo la posizione sociale. Si possono trovare denominazioni tecniche, mediche e numerosissime espressioni familiari (a volte anche volgari).

Il campo lessicale dipende dalle tradizioni culturali, dalla specificazione di un ambiente, dalla formazione e dalla personalità. Perciò ci sono notevoli differenze, $\mathrm{o}$ anzi un distacco insuperabile tra di loro, a tale punto che gli uni parlano un linguaggio "cortese" per esprimere i loro pensieri in modo sottile mentre il lessico degli altri è talvolta "osceno" per non dire volgare. La scelta di parole richiede l'improvvisazione, l'arte e l'intuito. Data la stratificazione per gruppi sociali, si può costatare che una parola non è quasi mai ugualmente interdetta con la stessa forza in tutti gli ambienti. Anche le classi più alte nell'uso colloquiale pronunciano i termini proibiti per educazione, soprattutto legati alla sfera sessuale. Inoltre le parole impronunciabili vengono ampiamente dette allo stesso modo da quelli che per norma d'educazione dovrebbero ometterle.

4. Per quanto riguarda il lessico "speciale" degli ambienti gerganti, come per esempio le parole militaresche o studentesche, esso assume la funzione di "controlingua" ed è un segno di riconoscimento sociopsicologico in una data società. Stabilizzando relazioni tra i membri all'interno del gruppo, il gergo difende nel contempo questo gruppo dagli altri, esprime la contrapposizione alla società e conferma la sua identità. In gergo si può parlare di tutto, ma viene usato più nei rapporti quotidiani fra cointeressati che in pubblico (Marcato, 1994: 761). Comunque la differenza essenziale tra l'uso delle parole gergali e quelle eufemistiche consiste nelle differenti cause psicologiche: dietro l'eufemismo sta l'interdizione, norma interiore nella maggioranza dei casi, invece alla base del gergo si trova l'esigenza pratica di non farsi capire (Galli de Paratesi, 1964: 10). Anche il cosidetto gruppo patologico costituito da parecchi degenerati tende necessariamente a creare modi speciali di relazionare. Nel linguaggio della malavita sono numerose le frasi o espressioni con rinvio alla vita amorosa che prima sono affettuose e poi diventano anche oscene, per esempio: ninnella, ninna, ninnola smilza, divia, confetta, miniera, gnifella per 'giovane bella'; terla, mignotta, galletto, ruscicarello per 'prostituta, civettuola'. Alla formazione di questa lingua oscena concorre la scarsezza di donne di facili costumi che si prostituiscono (Mirabella, 1910: 232). La funzione sociale, quella di affermare il senso di appartenenza al gruppo e di delimitazione del gruppo stesso verso l'esterno condivide certamente il linguaggio giovanile. In effetti esso ha molti tratti in comune con i gerghi, al punto di poter essere ritenuto una delle poche forme paragergali assai vitali nel repertorio italiano 
di oggi. E'indubbio che le caratteristiche interne del linguaggio giovanile, e alcune sue modalità d'uso, rivelino una componente di gioco e di scherzo che si realizza attraverso la deformazione della lingua. La sua grande creatività nasce dalla necessità di affermare e sottolineare l'immagine di sé (Cortelazzo, 1994: 294-295). Malgrado il frequente ricambio lessicale, vi si incontrano parole di lunga durata. Dietro di esse sta di solito una componente scherzosa (penna per 'ragazza', passera per 'organo maschile femminile') (ibidem: 309).

Poiché negli ultimi tempi si osserva che l'italiano evolve verso il basso le forme gergali e paragergali hanno avuto grande espansione in un primo tempo nel parlare scherzoso - familiare, assumendovi vari valori in rapporto alla complessività del dialogo, cioè espressivo, dissacrante o ammiccante. In seguito, molti di questi tratti prima caratteristici per gergalità o colloquialità (ma anche regionalità) sono stati catturati dalla norma e perdono così la loro marcatezza (Beccaria, 1988: 104).

5. Come si è già detto, spesso l'imbarazzo genera una perseveranza definitoria su ciò che non si può nominare, il desiderio di violare l'interdizione senza trasgredirla nettamente, il gusto di moltiplicare le allusioni rinunciando indefinitamente a pronunciare, per dire implicitamente. Il settore in cui si fa più ampio ricorso all'eufemismo è quello relativo al sesso per indicare quegli atti e quegli organi che il pudore impone di non chiamare con il proprio nome. Si nota anche il fatto che in quest'ambito è assai difficile indicare quale sia il vero nome di certe parti del corpo umano, giacché quello scientifico, proprio il lessico medico fornisce non numerosi esempi di sicuri eufemismi relativi alla sfera sessuale (tra poche eccezioni si nota utero), nella grande maggioranza dei casi è frutto di un'originaria metafora che si sta esaurendo o che più spesso autonomamente continua il proprio cammino attraverso la lingua, nei testi letterari e nei secoli. Né vi si può distinguere il caso delle salpingi che sono trombe bronzee, lunghe due metri, usate dai greci nelle cerimonie religiose e nelle azioni militari, la vagina è il fodero delle armi da taglio, il clitoride sembra in realtà provenire da una radice indoeuropea del significato originario di 'collinetta, rilievo'. Sono semplici ed evidenti i procedimenti di generazione metaforica che conducono all'attribuzione di un sovrasenso equivoco a una data espressione. Per esempio in inzuppare il biscottino nella tazza l'analogia della forma tra l'organo maschile e il dolce e l'organo femminile e il recipiente, l'atto di introdurre un corpo in un altro la presenza di liquidi durante il contatto tra i due oggetti, la sensazione di piacere (Boggione, Casalegno, 2000). Si nota comunque che il dolce, cioè qualcosa di buono, saporito, appartiene alla specie maschile, invece la specie femminile è paragonata ad un banale recipiente che potrebbe sembrare dispregiativo per una lettrice sensibile. Comunque ogni elemento della metafora svolge nella locuzione una funzione significativa; la sua forza espressiva dipende soprattutto dall'accordo interno del sistema lessicale e figurato, in cui è inclusa e dalla coerenza di questo sistema rispetto ai corrispondenti referenti. 
Si giunge alla costatazione che il lessico erotico è privo di intento attenuativo. In maniera mediata, attraverso un commento ironico e divertito, si intende far ridere, sorprendere, irritare e infatti non nascondere nulla. L'ambito lessicale erotico registra una prevalenza della connotazione sul valore denotativo, cerca di misurare la distanza che attraverso il gioco si è introdotta tra la cosa e l'espressione. Il linguaggio erotico non nasce dalla necessità di esprimere una cosa per cui manca il referente lessicale immediato o oggettivo, ma dal piacere e dal gusto di colorire e arricchire l'espressione con l'intento di stimolare nel lettore l'eccitazione, la meraviglia, la curiosità (Boggione, Casalengo, 2000). Attualmente si attribuisce al linguaggio erotico il rango di quello settoriale o anche specifico, e così il diritto di figurare nel proprio settore, nei dizionari ed enciclopedie, ai termini legati al mondo del sesso.

6. In italiano si notano anche le differenziazioni linguistiche correlate alla variabile sessuale. Esistono anche le differenze dovute alla diversa immagine presentata dai due sessi, al diverso ruolo dell'uomo e della donna nella società, al diverso tipo di socializzazione a cui vengono sottoposti tutte le componenti più culturali che sessuali (Berretta, 1983). Sono soprattutto i diversi atteggiamenti sociolinguistici che si dovrebbero evidenziare nelle varietà sociali legate al lesso, valutando ciò che caratterizza nell'ambito linguistico l'italiano parlato e scritto delle donne e quello degli uomini. Per quanto riguarda il lessico, alla lingua delle donne sono state attribuite preferenze per gli eufemismi, per le iperboli, per certi avverbi e modificatori, per i diminutivi e gli appellativi affettuosi (gioia, tesoro), per un vocabolario più ridotto, per un uso minore delle parole tabù (bestemmie, imprecazioni) e termini tecnici (Coveri, Benucci, Diadori, 1992: 105-107).

7. Ovviamente il settore del lessico erotico costituisce assai un campo di espressioni dai confini indefiniti e spesso incerti. In maggioranza le parole proprie di questo tipo di lessico costituiscono un uso estensivo di voci appartenenti non solo ad altri ambiti semantici, ma pure ad ambiti d'uso e di provenienza molto diversi, relative all'affettività e alla passione amorosa. I mezzi formali di cui dispone il sistema linguistico vengono realizzati nel discorso come eufemismi in vario modo figurati: metonimico (quello che si riferisce alla contiguità semantica) o metaforico (trasformazione del significato del sostituto in base alla similitudine). Il linguaggio amoroso include i termini di genere potenzialmente ambiguo o che si caratterizzano per una molteplicità di usi (aggettivi, avverbi o anche verbi usati come sostantivi), proviene dalle attività correnti, la vita domestica, il lavoro...

Il repertorio ha il numero incalcolabile di parole e di espressioni sostituive tanto che l'italiano evolve e si arricchisce continuamente di vocaboli nuovi. In genere si può sistematizzarli in voci relative:

a) alla donna: $b a b y$ (ragazza giovane donna), baccante (donna dominata da una forte eccitazione sessuale), bagascia (donna trasandata, di malaffare), bucal (brutta ragazza o in generale qualcosa di brutto), buzzona (donna dalla pancia voluminosa), cancello (ragazza bruttissima), chitarra (bella ragazza), ciabatta (persona spec. di 
sesso femminile, non più giovane $o$ in cattive condizioni di salute), cignala (ragazza), ciospa (ragazza di brutto aspetto), ciovetta (donna che fa di tutto per accentrare l'altrui attenzione su di sé), citofono (ragazza poco attraente), cozza (ragazza brutta), culona (persona con un sedere grosso), dondrona (donna grossa, pigra, lenta nei movimenti, svogliata), giallofia (donna giallastra, decrepita), goborchio (ragazza veramente brutta), gnocca (ragazza o donna molto vistosa e attraente), insperta (graziosa amatrice), lesbica, tribade (donna omossessuale), mastella (ragazza con grosse tette), frisa (una ragazza), gnappa, gnappetta (ragazza minuta e graziosa), vacca (donna di facili costumi oppure donna grassa e sfatta),

b) alla prostituzione: badia, casa, casa di tolleranza, casa chiusa, casa di appuntamento, casa squillo, casino, casotto, chiesa, monastero, osteria, postribolo (casa di prostituzione), badrusa (lucciola, donna di strada), bagascia (meretrice), brescialda (donna volgare, prostituta), colombina, figlia, figliola, gallinella, venere pandemia, libera pensatrice (prostituta), cortigiania (adulazione, prostituzione), gourmandise dei vecchi (prostituta), luci rosse (detto di tutto ciò che riguarda pomografia o prostituzione), mestiere (esercitare la prostituzione), fare la vita (esercitare la prostituzione),

c) all'organo femminile: baffa, gnocca, grotta, fessa, fica, fregna, frittella, parrucca, passera, passerina, patatina, potta (vulva), bernarda, caduca (decidua)', cazza, lallera, miciotta, patacca (vagina), conno (genitale esterno femminile), curcio, fessura, fiocca, mona, mussa, parte più delicata, patonza, scissura (organo genitale femminile), relitto (passera della vedova),

d) all'atto sessuale: boccaciccio (atto orale che la donna pratica con il genitale dell'uomo), concedere (darsi, spec. detto di donna che accetta un rapporto sessuale), concubito (il piacere insieme, spec. di uomo e donna, coito), congiungimento (unione sessuale, accoppiamento, raro unione matrimoniale), congiunzione, contatto, copula, copulazione (unione sessuale), cosare (avere rapporti sessuale), godere (provare l'orgasmo sessuale), gondone (profilattico, preservativo), guzzare (fare sesso), ditalino, venere solitaria (atto di masturbazione femminile), fare sesso (avere rapporti sessuali), trombata (rapporto sessuale), usamento (pratica sessuale),

e) alle parti del corpo: cinna, cioccia, ciuccia, poccia, poma, tetta, ubero, zinna, zizza (mammella, poppa), culo, fondoschiena, mappamondo, mazzo, messere, podice, preferito, sedici, tafanario (sedere, deretano, il posteriore),

f) alle funzioni e stati fisiologici: capoparto (prima mestruazione dopo il parto), malattie celtiche, mal francese (sifilide, mal francese), grossezza, gestazione, essere in stato interessato (pregnezza, gravidanza), interruzione (aborto), marchese (mestruo), menarca (comparsa della prima mestruazione nella pubertà), menorragia (mestruazione molto abbondante), menorrea (mestruazione), dismenorrea (mestruazione preceduta o accompagnata da dolore), dispareunia (dolore genitale avvertito soprattutto dalla donna durante il coito), frega (desiderio sessuale), una donna con il pancione (che è agli ultimi mesi di gravidanza), 
g) ai rapporti amichevoli o amorosi: ciuccione (bacio con la lingua), concitamento (eccitazione), corte, corteggiamento (lusingare per ottenere appoggi, favori, cercare di suscitare i sentimenti affettuosi, l'amore), dieta (astinenza sessuale), disamare (non amare più), disamoraggine (mancanza di amore / disamore), inciuccio (flirt), incugnare (fare l'amore), relazioni intime (relazione amorosa, sessuale), inversione sessuale, omofilia (omossessualità), love affair (relazione amorosa anche di breve durata), love story relazione amorosa, storia d'amore), lussuria (brama sfrenata di piaceri sessuali), fichi (carezze), fioccare (baciare, slinguazzare), foia (desiderio smodato, brama, frenesia), voglia (desiderio sessuale).

\section{CONCLUSIONI}

L'uso dei termini ed i loro sostituiti si confonde con un certo disagio nel pronunciarli e anche nello scriverli. Tale atteggiamento può dipendere da varie ragioni, spesso dal pudore, come nel caso di interdizione sessuale, soprattutto quando esso riguarda la femminilità. Per non offendere chi ascolta si tende a rispettare un codice che regola i rapporti con la società. Comunque, l'eufemismo tocca il linguaggio di tutte le classi sociali e si estende a tutti i livelli di stile, pes. familiare, letterario, confidenziale ecc...

In linea di massima si osservano le differenze tra il linguaggio femminile e quello maschile. Nonostante lo sviluppo sociale ed economico che permette alle donne di realizzarsi sul campo professionale, il lessico delle donne è considerato più conservatore. Anche la struttura sintattica comporta differenze: tende ad essere più semplice. Il loro linguaggio è affettivo e ricco di immagini in generale perché determinato da una parte dalle occupazioni con i bambini, cioè dalla formazione delle parole infantili e dal modo di parlare con essi. Le donne scelgono le sostituzioni a seconda del fatto che si rivolgono a se stesse o stanno in presenza di uomini, soprattutto per quando riguarda l'interdizione sessuale. In una conversazione tra donne invece di mestruazioni si useranno i sostituiti del tipo le mie cose o mal di pancia, di fronte ad uomini non se ne parlerà finchè possibile oppure si segnala un 'indisposizione o si dice è indisposta. Così le formule meno dirette ed evocatrici ossia i sostituiti alterati vengono favoriti quasi sempre dalle donne. Esse evitano di accennare gli organi sessuali e l'imprecazione sessuale è sconosciuta nel linguaggio femminile (Galli de Paratesi, 1964: 24-26).

Le parole che ruotano attorno al concetto della donna hanno subito lungo la loro storia numerosi cambiamenti di significato ed alcune innovazioni. Il concetto, di sesso e quindi del peccato, si lega ad essa oltre all'immagine dell'amore e dei sentimenti. Da una parte, sempre per ragioni sociali, si tende ad evitare un riferimento dirctto alla donna nella sua sessualità, i termini per "lei" rispecchiano il suo rango nella società e quelli che la confinerebbero ai livelli inferiori rientrano nel campo dell'eufemismo (ibidem: 77), per esempio: una serva non si usa più (è sconsigliata come parola brutta ricordante tristi leggi e costumi), anche al suo 
sostituito donna di servizio, donna si preferisce domestica o cameriera ed anzi persona di servizio; una donna significa 'una persona dall' aspetto socialmente modesto, si dice dunque preferibilmente una signora.

Dall'altra parte, soprattutto nei confronti con i "maschi", la lingua contiene delle sottigliezze che disprezzano incredibilmente le donne: alcune espressioni hanno un valore positivo se riguardano gli uomini, quando invece riguardano le donne sono negative, in questi esempi ogni denominazione femminile ha significato di 'una mignotta': una cortigiana, una massaggiatrice, una professionista, una donna di strada. una donna senza morale, una intrattenitrice, una donna pubblica. una donna facile, un'adescatrice, una donna molto disponibile ma al maschile significano rispettivamente un cortigiano 'uomo che vive a corte', un massaggiatore 'un Kinesiterapista', un professionista 'un uomo che conosce bene la sua professione', un uomo di strada 'un uomo duro', un uomo senza morale 'un politico', un intrattenitore 'un uomo socievole, affabulatore', un uomo pubblico 'un uomo famoso, in vista', un uomo facile 'un uomo con il quale è facile vivere', un adescatore 'un uomo che coglie al volo persone e situazioni', un uomo disponibile 'un uomo gentile'.

Per concludere questo breve studio si può costatare che comunque si trattano le donne con rispetto. Si attribuisce loro la massima espressione della sincerità, della sensibilità, della bellezza proseguendo la ricerca di spontaneità e freschezza linguistica, scegliendo i modi di dire che danno colore e vivacità al discorso.

\section{BIBLIOGRAFIA}

Beccaria G.L. (1988), Italiano antico e nuovo, Garzanti Editore, Milano.

Boggione W., Casalengo G. (2000), Dizionario letterario del lessico amoroso. Metafore, eufemismi, trivialismi, UTET, Torino.

Cortelazzo M. (1994), Il parlato giovanile, in Storia della lingua italiana, vol. II: Scritto e parlato, Giulio Einaudi Editore, Torino.

Cortelazzo M., Zolli P. (1990), Dizionario etimologico della tingua italiana, Zanichelli, Bologna.

Coveri L., Benucci A., Diadori P. (1992), Le varietà dell'italiano. Manuale di sociolinguistica italiana, Bonacci editore, Siena.

Galli de Paratesi N. (1964), Semantica dell'eufemismo. L'eufemismo e la repressione verbale con esempi tratti dall 'italiano contemporaneo, G. Giappichelli editore, Torino.

Marca to C. (1994), Il gergo, in Storia della lingua italiana, Vol. II: Scritto e parlato, Giulio Einaudi Editore, Torino.

Mirabella E. (1910), Mala vita. Gergo, camorra e costumi degli affiliati con 45000 voci della lingua furbesca in ordine alfabetico, Francesco Perrella Editore, Napoli.

Widlak S. (1970), Moyens euphémistiques en italien contemporain, Wydawnictwo Uniwersytetu Jagicllońskiego, Kraków.

Zing a relli N. (1998), Vocabolario della lingua italiana, Dodicesima edizione, Zanichelli, Bologna. 